\title{
Referências para ensinar Matemática Moderna no primário: o material Cuisenaire e a sistematização de saberes
}

\author{
References to teach New Mathematics in elementary school: \\ Cuisenaire's material and the systematization of knowledge
}

\section{Referencias para enseñar Matemática Moderna en la primaria: el material Cuisenaire y la sistematización de sabers}

\author{
Maria CRISTINA ARAÚJO DE OliveIRA (1 1 \\ Universidade Federal de Juiz de Fora, Juiz de Fora, MG, Brasil.
}

\begin{abstract}
RESUMO
O artigo apresenta um estudo sobre os saberes para ensinar matemática no ensino primário sistematizados em manuais pedagógicos que circularam em diferentes estados do Brasil nas décadas de 1960 e 1970. Particularmente, toma-se como foco o ensino de números e operações pelo método Cuisenaire. Nesse período se disseminaram propostas inovadoras relacionadas ao Movimento da Matemática Moderna. O texto orienta-se em torno do questionamento sobre a sistematização de saberes para ensinar matemática na perspectiva da Matemática Moderna, que se apresentava como referência para professores e normalistas, materializada em manuais pedagógicos. Esses saberes sistematizados, que dizem respeito à matemática para ensinar, um amálgama de conteúdo e métodos, uma criação voltada ao ensino primário, se constituem elementos de "profissionalidade" para professores primários. Assim, a "profissionalidade" é marcada pelo exercício de teorização pedagógica e didática que conduz a uma formalização da própria prática.

Palavras-chave: Método didático Cuisenaire. Matemática Moderna. Formação de professores. Normalistas.
\end{abstract}

\begin{abstract}
The article presents a study about the knowledge to teach mathematics in elementary school systematized in pedagogical manuals that circulated in different states of Brazil between the 1960s and 1970s. The main focus is in the teaching of numbers and operations by the Cuisenaire's method. During this period, innovative proposals related to the New Mathematics Movement were disseminated. It is oriented around the questioning about the systematization of knowledge to teach mathematics in this perspective, which was presented as a reference for elementary school teachers, materialized in pedagogical manuals. This systematized knowledge concerning mathematics to teach, an amalgam of content and methods, a creation aimed at elementary school, constitute elements of professionality for elementary school teachers. Thus, professionality is marked by the exercise of pedagogical and didactic theorizing, which leads to a formalization of the practice itself.
\end{abstract}

Keywords: Cuisenaire's method. New Mathematics. Professionality. Elementary school teachers.

\section{RESUMEN}

El artículo presenta un estudio sobre los saberes para enseñar matemática en enseñanza primaria sistematizados en manuales pedagógicos que circulaban en diferentes estados del Brasil entre las décadas de 1960 y 1970. Particularmente, se toma como eje la enseñanza de los números y operaciones por el método Cuisenaire. En este periodo se propagaran propuestas innovadoras relacionadas al movimiento de la matemática moderna. Se orienta alrededor del cuestionamiento sobre la sistematización de saberes para enseñar matemática en esta perspectiva, que se presentaba como referencia para profesores y normalistas, materializada en manuales pedagógicos. Estos saberes sistematizados que dicen respecto a la matemática para enseñar, una amalgama del contenido y métodos, una creación orientada a la enseñanza primaria, se constituyen en elementos de profesionalidad para profesores noveles. Así, la profesionalidad esta marcada por el ejercicio de teorización pedagógica y didáctica, que conduce a la formalización de la propia práctica.

Palabras clave: Método Cuisenaire. Matemática Moderna. Profesionalidad. Profesores noveles. 


\section{INTRODUÇÃO}

A constituição de saberes para o exercício da profissão docente tem sido objeto de investigações, discussões, proposições, teorizações desde pelo menos o século XIX. No Brasil, a institucionalização da instrução primária após a independência de Portugal implicou na problematização da formação dos professores primários, resultando na criação das escolas normais a partir de 1835, que passaram a ser, em uma trajetória histórica de altos e baixos, centros de referência para a formação dos professores primários - normalistas.

Esta breve e longínqua retrospectiva histórica pretende despertar para a rede de circunstâncias, contextos, possibilidades, produções materiais e bibliográficas que é requerida no processo de construção de saberes para ensinar, em particular, matemática.

Como determinados saberes e práticas para ensinar tornam-se perenes? Por que determinados conteúdos ou materiais se estabilizam na cultura escolar? O material de Cuisenaire ou reguinhas/barrinhas de Cuisenaire são um desses exemplos. Transformados ou apropriados em outras dimensões e materiais diferentes do proposto pelo autor Georges Cuisinaire Hottelet, esse material didático tem vida longa. A primeira publicação do criador sobre o material e as possibilidades de uso data de 1952. A partir daí se disseminou por vários países da Europa, Estados Unidos e América Latina, chegando ao Brasil na década de 1960.

Neste artigo, que investiga referências para ensinar matemática moderna no primário, problematiza-se o método de Cuisenaire, talvez mais conhecido pelo material didático do que propriamente pelo método que circulou no Brasil no período da Matemática Moderna (MM), entre as décadas de 1960 e 1980 . Cabe destacar que ainda hoje o material de Cuisenaire é indicado para o ensino dos números naturais e das frações, bem como das operações, além de noções de geometria ${ }^{1}$. Assim, investiga-se o percurso de sistematização e circulação do ensino de números e operações com o material Cuisenaire, ou com o método Cuisenaire, em uma perspectiva histórica.

\section{A CONSTITUIÇÃo DE UMA MATEMÁTICA PARA ENSINAR: A PROBLEMÁtica dOS SABERES}

Os saberes objeto de ensino - matérias, disciplinas, conteúdos - e os relativos ao modo como ensinar vêm

\footnotetext{
${ }^{1}$ O portal do professor no sítio do MEC disponibiliza plano de aula, referências bibliográficas e vídeos sobre o método Cuisenaire. Disponível em: http://portaldoprofessor.mec.gov.br/fichaTecnicaAula.html?aula=1563. Acesso em: 23 fev. 2019. Sítios de venda do material também apresentam breve descrição e possibilidades de aplicação para o ensino.
}

sendo estudados na sua relação com a formação dos professores desde a década de 1980 nos Estados Unidos. As pesquisas dedicam-se a investigar a formação de professores, seja continuada, inicial, em serviço. Muitos desses estudos se referem ao conhecimento prático pessoal, integrado na experiência e na prática de ensino. Os estudos de Goodson (2003) por meio da narrativa de história de vida se inserem nessa perspectiva, pela qual o saber não é concebido de forma diferenciada da ação. Barbier (1996) formula uma consequência teórica, a partir da distinção de duas significações da palavra saber: 1) o campo do "saber detido", que se inscreve na zona semântica das capacidades, dos conhecimentos, das competências, da disposição e das atitudes profissionais, que destaca como componentes identitários; e 2) o campo do "saber objetivado", que independe de quem os enuncia ou de quem deles se apropria. Os saberes objetivados são conserváveis, acumuláveis, apropriáveis. (BARBIER, 1996, apud HOFSTETTER; SCHNEUWLY, 2009, p. 17). Para o estudo histórico aqui apresentado interessou, particularmente, esse saber objetivado e o seu processo de objetivação ou sistematização. O saber detido pode ser identificado em cadernos e notas de professores, em projetos e materiais produzidos. Enquanto que o saber objetivado se materializa em normativas oficiais, revistas educacionais e manuais pedagógicos propostos aos professores.

Hofstetter e Schneuwly (2009) se distinguem das abordagens que adotam o ponto de vista da prática, considerando o saber a partir da sua mobilização no fazer. Os autores situam os saberes formalizados no centro de suas reflexões, tentando conceituar o seu papel na profissão docente. Dessa forma definem dois tipos constitutivos de saberes relativos à profissão docente: os saberes a ensinar, os saberes que são objeto do trabalho docente; e os saberes para ensinar, que são as ferramentas para a ação docente. Tais saberes não são tratados aqui isoladamente, mas como passíveis de serem distinguidos mesmo em sua estreita relação.

O conhecimento para a atuação docente, situado no campo da "profissionalidade", compreende o saber sobre: o assunto a ensinar, o estudante (em diferentes níveis, inclusive adultos), seus conhecimentos prévios, seu desenvolvimento, as formas de aprendizagem, as práticas de ensino (os métodos, os dispositivos, decomposição do saber a ensinar, modalidades de organização e de gestão, e outros).

A categorização de Shulman et al. (1987), frequentemente revisitada, compreende: o conhecimento dos conteúdos disciplinares, o saber pedagógico, o saber sobre o currículo, o saber sobre os aprendizes, o saber sobre os contextos educativos, o saber sobre as finalidades educativas e, sobretudo, o conhecimento pedagógico 
do conteúdo, um amálgama entre os conhecimentos do conteúdo e a pedagogia.

Uma perspectiva de "profissionalidade" pode ser então pensada como a construção do saber para ensinar a partir do saber a ensinar. Ao longo do processo de profissionalização da docência, um conjunto de saberes vão constituindo a "profissionalidade", saberes que são próprios ou compartilhados e participam da formação da identidade profissional "em função do público e do grau escolar, em função dos conteúdos e disciplinas de ensino, em função do vínculo institucional e do status profissional" (HOFSTETTER; SCHNEUWLY, 2009, p. 17).

Essa "profissionalidade" é marcada por um grande esforço de teorização pedagógica e didática, que implica também uma formalização mais ampla da própria prática. No contexto europeu, tanto os institutos de pesquisa quanto os de formação passaram por fundamentais transformações diante da necessidade de garantir uma nova articulação entre os saberes práticos/profissionais e os saberes científicos, entre o campo profissional e o campo disciplinar, fortalecendo as chamadas ciências da educação. No Brasil, o campo da Educação Matemática vem desempenhando esse papel, tomando para si a temática da formação dos professores que ensinam ou ensinaram matemática.

Analisando as relações entre os diferentes saberes presentes na formação, Hofstetter e Schneuwly (2009) apontam que nos níveis mais elementares os saberes são vistos como ferramentas para o ensino e, na medida em que os níveis de ensino crescem, eles vão assumindo o papel de objeto de ensino. Essa trajetória é acompanhada frequentemente do prestígio da categoria profissional, expresso pelo salário e pelo nível de formação. E ainda, a questão do gênero tem um papel significativo. As mulheres são maioria nos níveis mais elementares, enquanto que os homens, nos níveis mais escolarizados. Historicamente, são os professores primários os primeiros a reivindicarem os saberes para ensinar e a se beneficiarem nas instituições de formação dessa caracterização.

Considerando a especificidade da matemática e o contexto brasileiro, Valente (2017) analisa a constituição de uma matemática para ensinar no primário no final do século XIX que surge concomitantemente à criação dos grupos escolares, com aporte da pedagogia intuitiva e determina o declínio da referência à matemática do secundário para a formação dos professores primários. A introdução de novos suportes materiais na formação, como os cadernos de professores com as indicações didáticas para o ensino de matemática - aritmética, geometria -, mapas e materiais de ensino introduzem novos elementos na construção de saberes para ensinar matemática. Os materiais didáticos vão pouco a pouco estabilizando saberes para ensinar matemática em torno das possibilidades que conferem ao ensino de diversos conteúdos.

Os estudos em História da educação matemática nos mostram como determinadas práticas e saberes para ensinar matemática permanecem na cultura escolar, mesmo que sujeitos a frequentes mudanças nas pedagogias e nas finalidades da escola. A geometria euclidiana é um exemplo clássico (OLIVEIRA, 2018). A utilização do material de Cuisenaire para ensinar números (naturais, frações ou decimais) e as suas operações parece ser outro exemplo dessas permanências, que se verificam na cultura escolar, na matemática para ensinar. Arruda (2011) identifica vestígios de MM (Matemática Moderna) em sua prática docente na década de 1990, entre esses menciona a escala de Cuisenaire, uma outra maneira de se referir ao material.

\section{O MÉTOdo CUISENAIRE E A MATEMÁtica MODERNA NO ENSINO PRIMÁRIO}

Talvez no ensino primário tenham ocorrido as maiores transformações em torno de metodologias de ensino e processos de aprendizagem de matemática no contexto da Matemática Moderna. Nesse Movimento uma nova concepção de aprendizagem e consequentemente de ensino se estabeleceu, amparada em referências anteriores da escola nova, a partir da década de 1920, e em outros aportes, como os estudos de Piaget e a corrente estruturalista de pensamento.

Cabe também mencionar as aproximações dos matemáticos com a produção de saberes e práticas para a escola primária, buscando transformar conhecimentos matemáticos construídos em nível de pesquisa e de estruturação do campo científico, em matemática escolar. No Brasil, durante o processo de disseminação do MMM (Movimento da Matemática Moderna) na década de 1960, vários matemáticos desenvolveram atividades em grupos de estudos, como o Grupo de Estudos do Ensino da Matemática (GEEM) em São Paulo e outros; acompanharam a produção de livros didáticos e materiais para cursos de aperfeiçoamento ou capacitação em matemática, como eram denominados à época. Nomeadamente no ensino primário, o professor Jacy Monteiro, algebrista da Universidade de São Paulo (USP) foi supervisor da Coleção Curso Moderno de Matemática para o ensino de $1^{\circ}$ grau, que compreendia o atual Ensino Fundamental, produzida por um grupo de professoras: Anna Averbuch, Franca Cohen Gotlieb, Lucília Bechara Sanchez e Manhúcia Perelberg Liberman, em 1977. Formadas em Matemática, as professoras constituíram o Grupo de Ensino de Matemática Atualizada (GRUEMA), que assinava a Coleção (OLIVEIRA; LEME DA SILVA; VALENTE, 2011). 
No ensino primário a Matemática Moderna disposta em diferentes suportes, livros, manuais para professores, cursos de formação, impressos pedagógicos, e até mesmo Guias Curriculares, convocava a participação dos alunos na realização de atividades. Houve a promoção de cursos e o incentivo à utilização de materiais concretos como os blocos lógicos e o material de Cuisenaire. Destaca-se a diversidade de propostas metodológicas e de materiais para o ensino da Matemática Moderna no primário. Em especial, os jogos com base na lógica matemática, a manipulação de materiais didáticos para o estudo das operações e propriedades e, ainda, o uso dos livros didáticos. Nesse rol de propostas para a inclusão da Matemática Moderna para crianças, sobressaíram os seguintes materiais didáticos: os blocos lógicos de Zoltan Dienes, os blocos multibase, o material Cuisenaire e outros materiais não estruturados, como geoplano, cartões e tabelas.

Pela publicação que sistematizou os resultados de projeto de cooperação entre Brasil e Portugal sobre o MMM, (OLIVEIRA; LEME DA SILVA; VALENTE, 2011, p. 110), indica-se, que no ensino primário

vê-se a nítida preocupação dos formadores que atuavam junto aos professores primários, com a inovação das metodologias de ensino adequando-as à idade cognitiva das crianças. Ainda contemplando essas características etárias, investiu-se em impressos que atraíssem a atenção das crianças, como a inserção de representações pictóricas, cores e recursos gráficos, que até mesmo ultrapassaram os limites da tecnologia da época).

Nessa ambiência se insere a disseminação do método de Cuisenaire para a construção do conceito de número e o estudo das propriedades. Georges Cuisinaire Hottelet, educador belga, criou as barrinhas para ensinar os números, tornou pública a sua obra somente em 1952, primeira edição de Os números em cor: Novo Processo de Cálculo pelo Método Ativo, aplicável a todas as séries da Escola Primária. Após 23 anos de experiências com o uso desse material em escolas belgas, o contato com o educador Caleb Gattegno ${ }^{2}$ - egípcio radicado na Inglaterra - possibilitou a divulgação do material para diversos países, inclusive o Brasil, a partir da década de 1960. A produção de Gattegno voltou-se para publicações, palestras e cursos de Didática da Matemática. Ele teve

\footnotetext{
2 Caleb Gattegno (1911 - 1988) nasceu em Alexandria e morreu em Paris. Licenciado em Ciências em 1931 e Doutorado em Matemática em 1937, na Universidade de Basel. Mestre em Educação pela Universidade de Londres, em 1948, e Doutor em Filosofia pela Universidade de Lille em 1952. Foi um dos fundadores do International Commission for the Study and Improvement of Mathematics Education (CIEAEM) e, em 1952, da The Association for Teaching Aids in Mathematics (ATM) (SOUZA, 2005).
}

importante participação no Movimento de Matemática Moderna, principalmente no terreno da metodologia de ensino.

O método Cuisenaire foi estudado do ponto de vista psicopedagógico pelo Grupo Francês de Educação Nova (G.F.E.N.) entre 1958 e 1959. Com base na concepção de Piaget sobre a limitação da representação intuitiva para a compreensão da criança sobre a noção de número, o método enfatiza a ideia de conservação para o pensamento aritmético, sendo considerada uma espécie de a priori funcional desse. A manipulação das barrinhas permite verificar várias propriedades das operações (comutatividade, associatividade) e visualizar a realização das mesmas, inclusive conferir um tratamento de operador às frações.

Com base em pesquisas experimentais e argumentação apoiada em estudos psicológicos, se justificam as reguinhas ou barrinhas de Cuisenaire para o trabalho com a noção de conservação (de quantidades e de equivalências) central para a construção do número pelas crianças, na perspectiva piagetiana. O quadro da Figura 1 detalha as barrinhas a partir de suas funções para a exploração operatória.

Figura 1 - Quadro descritivo do material de Cuisenaire

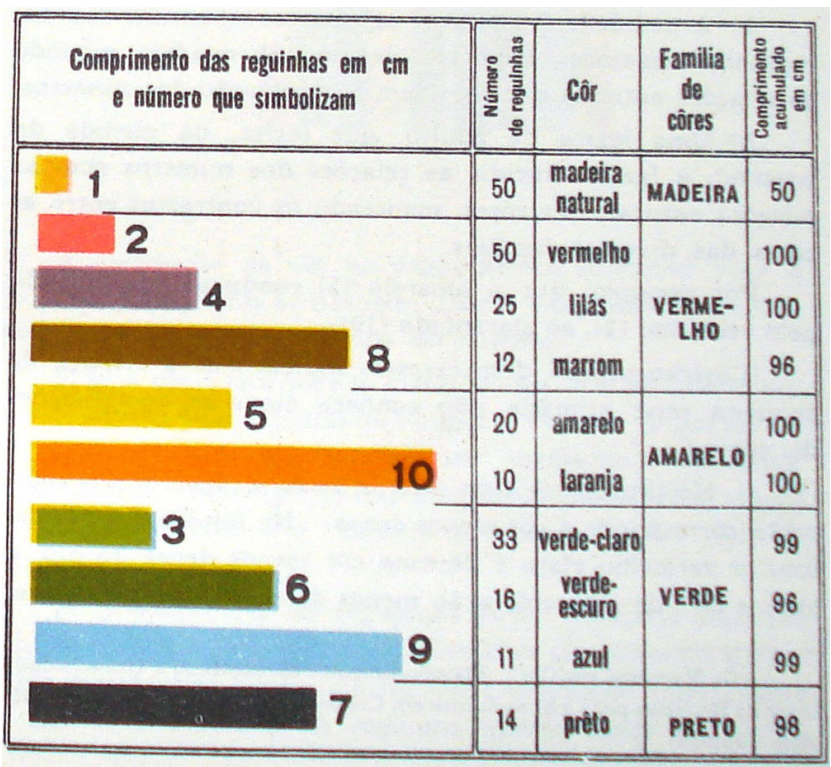

Fonte: Márquez, 1967, p. 61.

Por meio da manipulação das barrinhas, diferentes atividades podem ser realizadas para explorar a composição e decomposição de números naturais, fracionários ou em representação decimal. Pode-se também experimentar operações entre os números, visualizando os resultados.

A Figura 2 mostra como eram exemplificadas na obra de Márquez (1967) possíveis atividades envolvendo números e operações. 
Figura 2 - Representações de 12 com barrinhas Cuisenaire

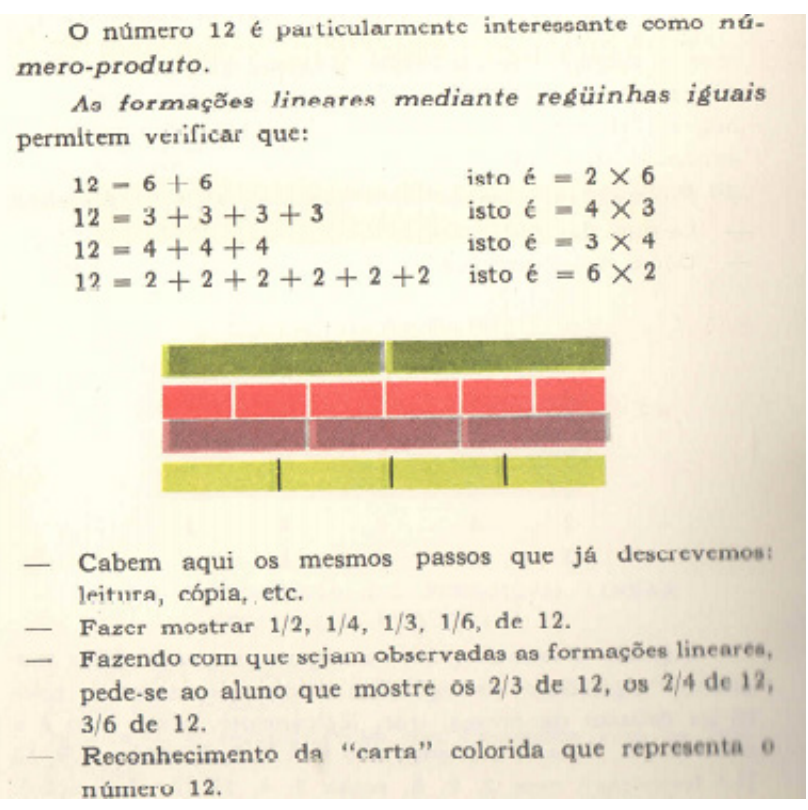

Fonte: Márquez, 1967, p. 124.

Nessa proposição é possível explorar diferentes decomposições do número 12 , em somas, produtos, mas também identificar frações, visualizando representações de frações de 12, por exemplo, a barrinha verde-claro que representa o número 3 é igual a 1/4 de 12 .

\section{BUSCANDO RASTROS DA CIRCULAÇÃo do método CuISENAIre no BRASIL}

Uma das publicações que participou desse processo de difusão do método em nível nacional foi o livro Didática das matemáticas elementares: o ensino das matemáticas pelo método dos números em côr ou método Cuisenaire, cujo autor é Ángel Diego Márquez ${ }^{3}$ (educador argentino), publicado inicialmente em 1964. No prólogo escrito por Manuel Santiago Rocca apresenta-se o autor como um divulgador de inovações, como o método Cuisinaire, mas que respeita e valoriza aspectos da pedagogia tradicional. Neste sentido, sobre o ensino de matemática afirma-se que para Ángel Diego Márquez as matemáticas "oferecem um instrumento prático sumamente valioso", mas também possuem "inegável valor formativo, permitindo uma valiosa ginástica intelectual, criando hábitos, fortalecendo a vontade e tornando ágil o raciocínio" (MÁRQUEZ, 1967, p.9). Assim, o educador concilia duas finalidades distintas da matemática na formação dos estudantes: atender às necessidades da vida cotidiana e formar $o$ espírito de modo a desenvolver o raciocínio em uma

\footnotetext{
${ }^{3}$ Doutor em Ciências Pedagógicas pela Universidade Livre de Bruxelas.
}

perspectiva mais propedêutica. Manuel Santiago Rocca, segundo escreve no prólogo da obra, foi testemunha da formação pedagógica de juventude de Diego Márquez na Escola Normal de professores Mariano Acosta - fundada em 1874 na cidade de Buenos Aires.

A tradução da obra para o português foi feita por Dirceu Accioly Lindoso 4 e foi publicada pela Editora Distribuidora de Livros Escolares LTDA. O exemplar disponível no repositório ${ }^{5}$ da UFSC foi localizado na biblioteca do grupo escolar Silviano Brandão, situado na cidade Silvianópolis - MG. Há também um exemplar no acervo do Laboratório de História da Educação Matemática (LaHem - UFJF), que pertenceu ao professor Ricardo Procópio, aposentado do Departamento de Matemática da UFJF.

Outro livro que apresenta sugestões de atividades para ensinar números e operações com o material de Cuisenaire é Didática Viva da Matemática no Curso Primário, de autoria de Maria Helena Roxo e Maria Luiza do Carmo Neves. A professora Maria Helena Roxo é apontada por Souza (2005) como introdutora do método Cuisenaire (também por propostas de Dienes e Montessori) na Coordenadoria de Ensino e Normas Pedagógicas do estado de São Paulo (CENP-SP). Já a professora Maria Luiza do Carmo Neves era licenciada em Matemática pela PUC-SP.

$\mathrm{Na}$ apresentação da obra, publicada em 1970, as professoras fazem referência à experiência que possuem no ensino com materiais como os blocos lógicos de Dienes e as barrinhas coloridas de Cuisenaire, desde 1967.

Figura 3 - Extrato da apresentação do livro Didática Viva da Matemática

Incluímos no trabalho exercicios e exemplos, utilizando-nos dos blocos lögicos de Dienes e das barminhas coloridas de Cuisenaire, recursos que estamos usando, intensivamente, desde 1967, com excelentes resultados.

Fonte: ROXO; NEVES, $1970^{6}$

As autoras destacam, como se pode ver no extrato anterior, os "excelentes resultados" no ensino de matemática a partir da utilização dos materiais didáticos mencionados. Na introdução assinada pelo professor Almerindo Marques Bastos ${ }^{7}$ a obra é enaltecida pelos

\footnotetext{
${ }^{4}$ Intelectual e jornalista ligado ao Partido Comunista no estado de Alagoas, participou da tradução de vários livros de Piaget junto com Rosa Maria Ribeiro da Silva.

5 Disponível em: https://repositorio.ufsc.br/handle/123456789/182516. Acesso em: 12 fev. 2019.

${ }^{6}$ Disponível em: https://repositorio.ufsc.br/handle/123456789/134539. Acesso em: 12 fev. 2019.

${ }^{7}$ Participou da elaboração do Guia Curricular de Matemática para o $1^{\circ}$ grau, publicado em 1973.
} 
anos de dedicação e trabalho das autoras em torno do ensino de matemática no primário.

O livro, composto de 13 capítulos, majoritariamente se organiza em torno de conteúdos - conjuntos, expressões, relações, numeração, geometria, problemas, entre outros - não se trata de um elenco usual de conteúdos simplesmente, mas de tópicos que têm forte apelo na Matemática Moderna, por exemplo, conjuntos e relações. Dois capítulos discutem particularmente as questões metodológicas: o primeiro, intitulado "A matemática no curso primário, escolha do material didático"; e o último, que trata dos materiais, blocos lógicos de Dienes e material Cuisenaire.

O capítulo inicial é breve, descreve o material Cuisenaire e recomenda o estudo de livros e a participação do professor em cursos especializados, bem como a discussão com colegas sobre o tema. Em nota de rodapé há a indicação do livro de Ángel Diego Márquez, apresentado anteriormente. No capítulo final, relativamente a esse material, são apresentadas atividades de composição e decomposição de números que se assemelham às propostas por Márquez (1967).

$\mathrm{Na}$ década de 1970, diferentes grupos de estudos do ensino de matemática, GEEM (MEDINA, 2007) em São Paulo, GEEMPA ${ }^{8}$ no Rio Grande do Sul (FISCHER; CARPES, 2007), NEDEM no Paraná (COSTA, 2013), desenvolveram cursos de formação para capacitar - termo usado à época - os professores para ensinar Matemática Moderna e os materiais que se associavam às propostas, destacadamente o material de Cuisenaire. Costa (2013), analisando a formação dos professores do estado do Paraná para ensinar Matemática Moderna, refere-se ao livro Didática Viva da Matemática no Curso Primário, anteriormente mencionado. Essa obra constava das referências de um manual com sugestões de atividades para os professores de $1^{\underline{a}}$ a $4^{\underline{a}}$ séries, correspondente ao ensino primário.

No Paraná os projetos de formação, capacitação, na década de 1970 divulgaram o uso de materiais didáticos para o ensino de matemática - entre eles o material Cuisenaire. Soares (2014) destaca a professora Maria Helena J. Reston Pinto como introdutora desse material, como professora de Metodologia de Matemática e Estágio Supervisionado no Instituto de Educação do Paraná, e posteriormente, como membro do NEDEM. Essa professora teria, anos mais tarde, retornado para o Rio Grande do Sul, o seu estado de origem, e participado do GEEMPA, afirmando, segundo Soares (2014), ter levado as suas experiências acumuladas para o grupo gaúcho.

\footnotetext{
8 GEEMPA - Grupo de Estudos sobre o Ensino de Matemática de Porto
} Alegre.
Medina (2007), analisando os Guias Curriculares de São Paulo, documento que orientou a educação paulista nas décadas de 1970 e 1980, oficializando a Matemática Moderna no estado, apresenta um quadro publicado na bibliografia dos Guias com onze possibilidades para o estudo de linguagem, codificação, conjuntos, relações, funções, contagem/numeração, operações (estruturas), equações, espaço, transformações, medidas. Para cada uma há várias sugestões de materiais didáticos; somente dois materiais, entre uma lista de vinte (que inclui blocos lógicos, blocos multibase, torre de Hanoi, entre outros), satisfazem a todas possibilidades, sendo um deles os números em cor Cuisenaire. Os Guias Curriculares são acompanhados de Subsídios para o $1^{\mathrm{O}}$ grau, publicados em 1975, que orientam como o professor poderia conduzir as aulas introduzindo novos conteúdos e materiais concretos. Para a efetiva implementação da proposta dos Guias, o estado promoveu cursos de treinamento para os professores da rede ao longo das décadas de 1970 e 1980.

Borges (2017) menciona a disseminação do material de Cuisenaire em Portugal por meio de adaptação realizada pelo professor João Antônio Nabais, considerado o introdutor da metodologia dos cubos - barras de cor para a aprendizagem matemática em Portugal - e a realização de cursos para a utilização deste e de outros materiais didáticos no contexto da Matemática Moderna.

\section{O material Cuisenaire e a SISTEMATIZAÇÃo DE SABERES PARA ENSINAR MATEMÁTICA}

Para arrematar este texto retomamos o título do artigo, que destaca as referências para ensinar Matemática Moderna no primário, tomando como centro o material Cuisenaire. A análise histórica da sistematização de um saber para ensinar números e operações com esse material nos permite perceber como, por meio de livros para professores, cursos de capacitação (na nomenclatura da época do MMM) ou formação de professores, apostilas e outros suportes materiais, além das relações pessoais, vão se disseminando e institucionalizando saberes para ensinar matemática.

O método Cuisenaire pensado em uma perspectiva mais generalista, ou seja, materializado na utilização do material para a construção do conceito de número e o estudo das operações, é ainda utilizado, inclusive com orientações no Portal do Professor no sítio do MEC. Tal permanência é indicativa de que a proposta tem sido absorvida pela cultura escolar, com as devidas transformações requeridas em cada época por esta própria cultura. Outros materiais vieram, como o Frac-Soma 235, criado pelo professor Roberto Baldino, em 1984, composto de peças que diferem em comprimento e cor 
e se assemelham as do material Cuisenaire. Contudo, as atividades associadas a este material têm natureza distinta, caberia investigação mais aprofundada sobre isso.

Os saberes sistematizados em livros, cursos de formação e impressos educacionais vão constituindo a profissionalidade dos professores primários para o trabalho com a matemática escolar. Destacadamente, o uso de material concreto, aos moldes das barrinhas Cuisenaire, introduziu uma nova prática para ensinar e possibilitar a construção do conceito de número pelas crianças. A possibilidade de manipular, experimentar, concretizar e validar hipóteses parece conferir ao material a sua longevidade.

\section{REFERÊNCIAS}

ARRUDA, J. P. Histórias e práticas de um ensino na escola primária: marcas e movimentos da matemática moderna. 2011. Tese (Doutorado em Educação Cientifica e Tecnológica) - Universidade Federal de Santa Catarina, Florianópolis, 2011. https://doi.org/10.20873/UFt.2525-4863.2017v2n3p965

BARBIER, J. (ed.). Savoirs théoriques et saviors d'action. Paris: PUF, 1996.

BORGES, R. A. S. O caderno do professor Moreirinhas Pinheiro e a matemática na escola de magistério primário de Lisboa (década de 1960.) In: SEMINÁRIO TEMÁTICO "CADERNOS ESCOLARES DE ALUNOS E PROFESSORES E A HISTÓRIA DA EDUCAÇÃO MATEMÁTICA, 1890-1990”, 15., 2017, Pelotas. Anais [...]. Pelotas: Universidade Federal de Pelotas, 2017. v. 1. p. 1-18. https://doi.org/10.5327/z2176-947820170233

COSTA, R. R. O aperfeiçoamento de professores em matemática nas décadas de 1960 e 1970 no estado do Paraná. 2013. Tese (Doutorado em Educação) - Pontifícia Universidade Católica do Paraná, Curitiba, 2013. https://doi. org/10.5327/z2447-211520181700087

FISCHER, M. C. B.; CARPES, F. Reformulação metodológica do ensino da matemática no $1^{\circ}$ grau: análise preliminar do relatório de pesquisa realizada pelo GEEMPA (1975). In: MATOS, José Manuel; VALENTE, Wagner Rodrigues (org.). A Matemática Moderna nas escolas do Brasil e de Portugal: primeiros estudos. São Paulo: Da Vinci, 2007. p. 123- 135. https://doi.org/10.11606/t.48.2012.tde14052013-103937

GOODSON, I. F. Professional knowledge, professional lives: studies in education and change. Maideanhead: Open University Press, 2003.

HOFSTETTER, R.; SCHNEUWLY, B. Savoirs en (trans)formation: au coeur des professions de l'enseignement et de la formation. Bruxelas: De Boeck, 2009. https://doi. org/10.3917/dbu.hofst.2009.01.0007

MÁRQUEZ, A. D. Didática das matemáticas elementares: o ensino das matemáticas pelo método dos números em côr ou método Cuisenaire. Rio de Janeiro: Distribuidora de Livros Escolares, 1967.
MEDINA, D. A produção oficial do MMM para o ensino primário do Estado de São Paulo (1960-1980). 2007.

Dissertação (Mestrado em Educação Matemática) - Pontifícia Universidade Católica de São Paulo, São Paulo, 2007. https://doi.org/10.29289/259453942018v28s1057

OLIVEIRA, M. C. A. Percurso investigativo sobre a geometria e o desenho como saberes profissionais de professores dos anos iniciais no Brasil. Paradigma, Maracay, v. XXXIX, p. 175-189, 2018.

OLIVEIRA, M. C. A.; LEME DA SILVA, M. C. L.; VALENTE, W. R. (org.). O Movimento da Matemática Moderna: história de uma revolução curricular. Juiz de Fora: Editora UFJF, 2011. v. 1. 190 p. https://doi.org/10.7213/rde. v6i18.3235

ROXO, M. H.; NEVES, M. L. C. Didática viva da Matemática no Curso Primário. São Paulo: Moderna, 1970.

SHULMAN, L. S. et al. 150 ways of knowing: Representations of knowledge in teaching. In: CALDERHEAD, J. (org.). Exploring teachers thinking. Grã-Bretanha: Cassel Educational Limited, 1987. p. 104-124.

SOARES, E. T. P. Zoltan Paul Dienes e o sistema de numeração decimal na cultura escolar paranaense (1960-1989). 2014. Tese (Doutorado em Educação) Pontifícia Universidade Católica do Paraná, Curitiba, 2014.

SOUZA, G. L. D. Educação Matemática na CENP: um estudo histórico sobre condições institucionais de produção cultural por parte de uma comunidade de prática. 2005. Tese (Doutorado em Educação) - Universidade Estadual de Campinas, Campinas, 2005. https://doi.org/10.17143/ciaed/ xxiilciaed.2017.00435

VALENTE, W. R. Dos livros didáticos para os cadernos de matemática: a emergência dos saberes profissionais. Zetetiké (on line), Campinas, v. 25, p. 254, 2017. https://doi. org/10.20396/zet.v25i2.8648206

Recebido em: 1/4/2019.

Aprovado em: 14/5/2019.

Publicado em: 8/11/2019.

Endereço para correspondência:

Maria Cristina Araújo de Oliveira

Universidade Federal de Juiz de Fora

Departamento de Matemática - Instituto de Ciências Exatas

Campus Universitário

Rua José Lourenço Kelmer, s/n - Bairro São Pedro

36036-900, Juiz de Fora, MG, Brasil

Autora:

Maria CRistina AraúJo de OliveIRa

Doutora em Educação pela Pontifícia Universidade Católica de São Paulo

(PUC-SP), São Paulo, SP, Brasil. Mestra em Matemática pela Universidade de São Paulo (USP), São Paulo (SP), Brasil. Professora Associada do Departamento de Matemática do Instituto de Ciências Exatas e do Programa de Pós-graduação em Educação Matemática da Universidade Federal de Juiz de Fora (UFJF),

Juiz de Fora, MG, Brasil. Pesquisadora do GHEMAT-Brasil.

Orcid: http://orcid.org/0000-0003-3346-1578

E-mail: mcrisoliveira6@gmail.com 